\title{
Finite Element Method for Internal Wave Equation for Stratified Fluid
}

\author{
Dawletbay Utebaev ${ }^{1}$ \\ ${ }^{1}$ Kara-Kalpak Berdakh State University, Abdirov, Uzbekistan \\ Correspondence: Dawletbay Utebaev, Kara-Kalpak Berdakh State University, Abdirov st. 1, Nukus 230100, \\ Uzbekistan. E-mail: dutebaev56@mail.ru
}

Received: July 9, 2012 Accepted: July 23, 2012 Online Published: August 20, 2012

doi:10.5539/cis.v5n5p88 URL: http://dx.doi.org/10.5539/cis.v5n5p88

\begin{abstract}
In many areas of natural science non-stationary problems on internal wave motion arise. For example, such problems appear in aerophysics, geophysics, oceanology, in theory of rotating fluid, and at design and construction of mining constructions (Gabov \& Sveshnikov, 1990). It is a complicated mathematical problem to obtain accurate analytical solutions for such problems. In such cases a natural apparatus for study of internal wave motion processes is in numerical methods. In this work, the schemes of the finite element method with high accuracy in space and time for solution of a mixed boundary problem for internal wave equation for stratified fluid are proposed and studied. The schemes constructed have specific advantages compared to the other schemes: a scheme with high order of accuracy (more than two); besides the solution itself one finds along which that its derivative (velocity) with the same accuracy; at use of interpolation representation of the solution one can obtain, if needed, the solution and its derivative for an arbitrary instant; since the schemes are two-layer ones, one can use variable step without loss of accuracy; the scheme is conventionally stable and requires 4 times more arithmetical operations compared to ordinary ones, though this scheme makes it possible to choose larger time steps to get given accuracy. Besides that, evaluations of accuracy of the schemes for the problem under consideration are obtained. By means of dispersion analysis comparison to known schemes is carried out.
\end{abstract}

Keywords: finite-element method, difference scheme, estimates of accuracy, dispersion analysis

\section{Problem Statement}

Let us consider a problem (Gabov \& Sveshnikov, 1990)

$$
\begin{gathered}
\frac{\partial^{2}}{\partial t^{2}}\left(L_{1} u-\beta^{2} u\right)+L_{2} u=f(x, t),(x, t) \in Q_{T}=\{x \in \Omega, t \in(0, T]\}, \\
u=0, \quad x \in \Gamma_{1} ; \quad \frac{\partial u}{\partial n}=0, x \in \Gamma_{2} ; t \in[0, T], u(x, 0)=u_{0}(x), \quad \frac{\partial u}{\partial t}(x, 0)=u_{1}(x) .
\end{gathered}
$$

Here $L_{1} u=\Delta_{2} u \equiv \frac{\partial^{2} u}{\partial x_{1}^{2}}+\frac{\partial^{2} u}{\partial x_{2}^{2}}, L_{2} u=\omega_{0}^{2} \Delta_{1} u \equiv \omega_{0}^{2} \frac{\partial^{2} u}{\partial x_{1}^{2}} ; \omega_{0}$ - Väisälä-Brunt frequency; $\Omega=\left\{x=\left(x_{1}, x_{2}\right): 0<x_{\alpha}<\right.$ $\left.l_{\alpha}, \alpha=1,2\right\}, \Gamma_{1} \cup \Gamma_{2}=\Gamma=\partial \Omega ; \beta$ - parameter that characterizes stratification of fluid.

Let us identify as generalized solution of the problem (1) the function $u(x, t)$ that at each $t \in(0, T]$ belongs to space $H=\stackrel{\circ}{W}_{2}^{1}(\Omega)$, has a derivative $\frac{\partial^{2} u}{\partial t^{2}} \in W_{2}^{1}(\Omega)$ and almost everywhere for all the $t \in(0, T]$ obeys the relationships $\forall \vartheta(x) \in H$ :

$$
a_{1}\left(\frac{d^{2} u(t)}{d t^{2}}, \vartheta\right)+a_{2}(u(t), \vartheta)=(f(t), \vartheta), \quad u(0)=u_{0}, \quad \frac{d u}{d t}(0)=u_{1}, \quad \forall \vartheta(x) \in H .
$$

Here $a_{1}(u(t), \vartheta)=\int_{\Omega}\left(\frac{\partial u}{\partial x_{1}} \frac{\partial \vartheta}{\partial x_{1}}+\frac{\partial u}{\partial x_{2}} \frac{\partial \vartheta}{\partial x_{2}}-\beta^{2} u \vartheta\right) d x, a_{2}(u(t), \vartheta)=\omega_{0}^{2} \int_{\Omega} \frac{\partial u}{\partial x_{1}} \frac{\partial \vartheta}{\partial x_{1}} d x$. Let us denote as $|u|_{m}=\sqrt{a_{m}(u, u)}$, $m=1,2$, the energetic semi-norms in $H$ that correspond to bilinear forms $a_{m}(u, \vartheta)$. The energetic space $H_{A_{1}}$, generated by a semi-norm $|u|_{1}$, is equivalent to space $H=\stackrel{\circ}{W} \underset{2}{1}(\Omega)$, so estimations $c_{1}\|u\|_{1}^{2} \leqslant a_{1}(u, u) \leqslant C_{1}\|u\|_{1}^{2}$ and $0 \leqslant a_{2}(u, u) \leqslant C_{2}\|u\|_{1}^{2}$ are valid. Here $c_{1}, C_{1}, C_{2}$ - positive constants that depend on $\beta, \omega_{0}$. 
The existence, continuity, and uniqueness of the solution of this problem are discussed in (Gabov \& Sveshnikov, 1990).

\section{Discretization by Space and Time}

Let us perform discretization of the problem by spatial variables by means of the finite element method. Let $H_{h} \subset H$ is a set of elements of the type $\vartheta_{h}=\sum_{i=1}^{N} a_{i} \varphi_{i}(x)$. Here $\left\{\varphi_{i}=\varphi_{i}(x)\right\}_{i=1}^{N}$ is a basis of piecewise-polynomial functions that are polynomials of power $k$ at each finite element (Quarteroni \& Valli, 1994).

Let us bring into correspondence with (2) a semi-discrete problem for $t \in[0, T]$ :

$$
a_{1}\left(\frac{d^{2} u_{h}(t)}{d t^{2}}, \vartheta_{h}\right)+a_{2}\left(u_{h}, \vartheta_{h}\right)=\left(f(t), \vartheta_{h}\right), u_{h}(0)=u_{0,1}, \frac{d u_{h}}{d t}(0)=u_{1, h}, \quad \forall \vartheta_{h} \in H_{h}
$$

To the problem (3) a Cauchy problem corresponds:

$$
D \frac{d^{2} u_{h}(t)}{d t^{2}}+A u_{h}(t)=f_{h}(t), \quad u_{h}(0)=u_{0, h}, \frac{d u_{h}}{d t}(0)=u_{1, h} .
$$

Operators $D, A$ act from $H_{h}$ to $H_{h}$. To those rigidity matrices $\mathbf{D}=\left(a_{1}\left(\varphi_{i}, \varphi_{j}\right)\right)_{i, j=1}^{N}$ and $\mathbf{A}=\left(a_{2}\left(\varphi_{i}, \varphi_{j}\right)\right)_{i, j=1}^{N}$ correspond. Here $u_{0, h}=u_{I}(0)$ - interpolant of initial value of the solution $u_{I}(0)=P_{h} u_{0}(x)$, and $P_{h}$ - projection operator $P_{h}: H \rightarrow H_{h}$.

Let us approximate the problem (4) with a two-parameter difference scheme obtained by the finite element method in time (Moskal'kov, 1980; Moskalkov \& Utebaev, 2005):

$$
\begin{gathered}
\left(D-\frac{\tau^{2}}{12} A\right) \frac{\hat{y}-\dot{y}}{\tau}+A \frac{\hat{y}+y}{2}=\varphi_{1},\left(D-\alpha \tau^{2} A\right) \frac{\hat{y}-y}{\tau}-\left(D-\beta \tau^{2} A\right) \frac{\hat{y}+\dot{y}}{2}=\varphi_{2}, \\
y^{0}=u_{0}, \dot{y}^{0}=u_{1} .
\end{gathered}
$$

Here it is denoted: $y=y^{n}=y\left(t_{n}\right), \hat{y}=y^{n+1}, \dot{y}=\dot{y}^{n}=\frac{d y}{d t}\left(t_{n}\right), n=0,1, \ldots, y^{n}, \dot{y}^{n} \in H_{h}$. Then, $\varphi_{k}=\int_{0}^{1} f\left(t_{n}+\tau \xi\right) \vartheta_{k}(\xi) d \xi$, $k=1,2, \xi=\left(t-t_{n}\right) / \tau, \vartheta_{1}(\xi)=1, \vartheta_{2}(\xi)=s_{1} \vartheta_{2}^{(1)}(\xi)+s_{2} \vartheta_{2}^{(2)}(\xi), \vartheta_{2}^{(1)}(\xi)=\tau(\xi-1 / 2), \vartheta_{2}^{(2)}(\xi)=\tau\left(\xi^{3}-3 \xi^{2} / 2+\xi / 2\right)$, $s_{1}=180 \beta-40 \alpha, s_{2}=1680 \beta-280 \alpha$.

The solution looks like a Hermite polynomial that interpolates $y^{n}, y^{n+1}, \dot{y}^{n}, \dot{y}^{n+1}$ at interval $t \in\left(t_{n}, t_{n+1}\right)$ :

$$
y(t)=y^{n} \varphi_{00}^{n}(t)+\dot{y}^{n} \varphi_{10}^{n}(t)+y^{n+1} \varphi_{01}^{n}(t)+\dot{y}^{n+1} \varphi_{11}^{n}(t), t \in\left(t_{n}, t_{n+1}\right), n=0,1, \ldots,
$$

where $\varphi_{00}^{n}(t)=2 \xi^{3}-3 \xi^{2}+1, \varphi_{01}^{n}(t)=3 \xi^{2}-2 \xi^{3}, \varphi_{10}^{n}(t)=\tau\left(\xi^{3}-2 \xi^{2}+\xi\right), \varphi_{11}^{n}(t)=\tau\left(\xi^{3}-\xi^{2}\right)$. For $t \in[0, T]$ - this is a Hermite spline of 3-d power (Declu, 1976).

\section{Theorems on Accuracy}

Let us formulate the result on stability and accuracy of the scheme (5), (6) (Moskal'kov, 1980).

Theorem 1. If $A^{*}=A>0, D^{*}=D>0$ and

$$
\alpha-\beta=1 / 12, D-\delta \tau^{2} A \geqslant \varepsilon D, 0<\varepsilon<1, \delta=\max \{\alpha, \beta, 1 / 12,0\},
$$

solution $y(t)$ of the scheme (5), (6) converges to solution of the problem (4) $u_{h}(t) \in C^{6}[0, T]$ and evaluation

$$
\left\|u_{h}(t)-y(t)\right\|_{A}+\left\|\dot{u}_{h}(t)-\dot{y}(t)\right\|_{D} \leqslant M \tau^{4}
$$

is valid.

The proof is based on bringing the two-layer vector scheme (5), (6) to a three-layer one, separately for the solution $y$ and its derivative $\dot{y}$. Condition (8) for values of parameters $\alpha=1 / 8, \beta=1 / 24$, and thus $\delta=1 / 8$, leads to such a restriction of step in time: $\tau \leqslant 2 \sqrt{2} /\left[\omega_{0}(1-\varepsilon)\right]$ - condition of stability of the scheme. 
To evaluate accuracy of the scheme one has to estimate the error $z=u_{h}-u$. With use of the method of such estimation in the theory of difference schemes (Samarskii, 1977) and the theory of finite element method (Quarteroni $\&$ Valli, 1994) we formulate the following result.

Theorem 2. Let conditions of the theorem 1 are satisfied. Then the solution for the scheme (5), (6) converges to a smooth enough solution of the problem (1), and estimation

$$
\|u(x, t)-y(x, t)\|_{1}+\|\dot{u}(x, t)-\dot{y}(x, t)\|_{1} \leqslant M\left(h^{3}+\tau^{4}\right)
$$

is valid. With application of a special technology for evaluation of accuracy by space and time, one can decrease requirements for smoothness of the solution $u(x, t)$. Following statement takes place (Moskalkov \& Utebaev, 2011a).

Theorem 3. Let conditions of the theorem 1 are satisfied. Then for such a solution for the scheme (5), (6) approximating the solution of the problem (1) that $u(x, t), \frac{\partial u}{\partial t}(x, t) \in C\left([0, T] ; W_{2}^{k+1}(\Omega) \cap \stackrel{\circ}{W} \underset{2}{1}(\Omega)\right), \frac{\partial^{4} u}{\partial t^{4}}(x, t) \in$ $C\left\{[0, T] ; W_{2}^{2}(\Omega)\right\}$, following evaluation of accuracy is valid:

$$
\begin{gathered}
\|u(x, t)-y(x, t)\|_{1} \leqslant M\left\{h^{k}\left(\max _{t}\left\|\frac{\partial u}{\partial t}(x, t)\right\|_{k+1}+\sqrt{\int_{0}^{t}\left\|u\left(x, t^{\prime}\right)\right\|_{k+1}^{2} d t^{\prime}}\right)+\right. \\
\left.+\tau^{3} \sqrt{\int_{0}^{t}\left\|\frac{\partial^{4} u}{\partial t^{4}}\left(x, t^{\prime}\right)\right\|_{2}^{2} d t^{\prime}}\right\} \forall t \in[0, T], M=M\left(T, \beta, \omega_{0}^{2}\right)>0 .
\end{gathered}
$$

At choosing at each finite element, by space of polynomial, of degree not lower than the third one, we have the third order of accuracy by both steps $h, \tau$.

\section{Analysis of Dispersion of Difference Schemes}

The Equation (1) relates to equations with strong dispersion. Dispersion equation for solutions of harmonic type

$$
u=\exp \left[i\left(\omega t+k_{1} x_{1}+k_{2} x_{2}\right)\right], i^{2}=-1,
$$

gives the following frequency value

$$
\omega= \pm \omega_{0} k_{1} / \sqrt{k_{1}^{2}+k_{2}^{2}+\beta^{2}} .
$$

Phase velocity depends on the components of the wave vector $\vec{k}=\left(k_{1}, k_{2}\right)$ :

$$
v=\omega / \sqrt{k_{1}^{2}+k_{2}^{2}}= \pm \omega_{0} k_{1} / \sqrt{\left(k_{1}^{2}+k_{2}^{2}\right)^{2}+\beta^{2}\left(k_{1}^{2}+k_{2}^{2}\right)}
$$

and is evidence of decrease of phase velocity of harmonics with increase of $k_{1}, k_{2}$. For uneven solutions of the equation, harmonics with higher values of components of the wave vector $\vec{k}=\left(k_{1}, k_{2}\right)$ make a substantial input to solution of the problem (1). Let us consider how harmonics are transferred in numerical solution. To simplify the study, let us consider a uniform test equation:

$$
\ddot{u}+\omega^{2} u=0 .
$$

Here frequency $\omega$ corresponds to (9). Accurate solution (10):

$$
u(t)=a_{1} \cos \omega t+a_{2} \sin \omega t .
$$

Constant parameters $a_{1}, a_{2}$ from (11) are determined by initial conditions.

We look for the solution for difference Equations (5) $\left(\varphi_{1}=\varphi_{2}=0\right)$ that correspond to (9) in form $y=y^{n}=$ $Y q^{n}, \quad \dot{y}=\dot{y}^{n}=\dot{Y} q^{n}$ with amplitudes $Y$ and $\dot{Y}$. At satisfaction of stability condition $\tau^{2} \leqslant 1 /\left(m \omega^{2}\right), \quad m=$ $\max \{\alpha, \beta, \gamma, 0\}>0$ we have

$$
y=y^{n}=b_{1} \cos \frac{\varphi}{\tau} t_{n}+b_{2} \sin \frac{\varphi}{\tau} t_{n},
$$

where $\varphi=2 \arcsin \left[\frac{z}{2} \sqrt{\left(1-\beta z^{2}\right) /\left(\left(1-\alpha z^{2}\right)\left(1-\gamma z^{2}\right)+\frac{z^{2}}{4}\left(1-\beta z^{2}\right)\right)}\right]$. Here $\gamma=1 / 12, z=\omega \tau$. 
Difference for the accurate and approximate solutions one can characterize by value $\delta \omega=\varphi / \tau \omega$ - relative error of frequency of oscillations $\bar{\omega}=\varphi / \tau$ for the difference Equation (5). At $\delta \omega$ closer to 1, more accurate approximate solution is.

Let us expand $\delta \omega$ in series by powers of $z^{2}$ :

$$
\begin{gathered}
\delta \omega=1+r_{1} z^{2}+r_{2} z^{4}+O\left(z^{6}\right), \\
r_{1}=(\alpha+\gamma-\beta-1 / 6) / 2, \quad r_{2}=(\beta-6 \alpha \gamma+1 / 40) / 12+(\alpha+\gamma-\beta-1 / 6) \bar{r}_{2} .
\end{gathered}
$$

With minimization of $\left|r_{1}\right|$ and $\left|r_{2}\right|$, one can improve quality of approximate solution. For all the schemes considered further we require satisfaction of the condition $r_{1}=0$. Hence

$$
\alpha+\gamma=\beta+1 / 6
$$

At that $\delta \omega=1+r_{2} \omega^{4} \tau^{4}+O\left(\omega^{6} \tau^{6}\right), r_{2}=(\beta-6 \alpha \gamma+1 / 40) / 12$. In this case one can say of coincidence of velocity of propagation of harmonics of differential equation and difference scheme with accuracy up to values of fourth order by step $\tau$. It is obvious that at that estimation of error of the solution of Equation (4) $z=u(t)-y(t)=O\left(\tau^{4}\right)$ takes place.

Examples of schemes with fourth order of accuracy are the schemes (5), (6) with parameters:

(1) $\gamma=1 / 12, \alpha=1 / 8, \beta=1 / 24$ and stability condition $\tau^{2} \leqslant 8 / \omega^{2}$;

(2) $\gamma=1 / 12, \alpha=1 / 10, \beta=1 / 60$ and stability condition $\tau^{2} \leqslant 10 / \omega^{2}$. Let us give one more example of a scheme with sixth order of accuracy $\left(r_{2}=0\right)$ from this class;

(3) $\gamma=0.302705, \alpha=0.197295, \beta=1 / 3$. Stability condition $\tau^{2} \leqslant 3 / \omega^{2}$. Let us compare those to the known Numerov's scheme of the 4-th order of accuracy (Bakhvalov, Zhidkov, \& Kobelkov, 1987). For the problem (4) it looks like

$$
\left(D+\tau^{2} A / 12\right) y_{\bar{t} t}+A y=\varphi .
$$

As applied to the Equation (10) this leads to

$$
\left(1+\tau^{2} \omega^{2} / 12\right)\left(y^{n+1}-2 y^{n}+y^{n-1}\right) / \tau^{2}+\omega^{2} y=0 .
$$

For solution of type $y^{n}=q^{n}$ we have a characteristic equation

$$
q^{2}-2(1-\mu / 2) q+1=0, \mu=\omega^{2} \tau^{2} /\left(1+\omega^{2} \tau^{2} / 12\right)
$$

For $\mu \leqslant 4$ the roots (13) are: $q_{1,2}=\cos \varphi \pm i \sin \varphi$, where $\cos \varphi=1-\mu / 2$. Hence $\varphi=2 \arcsin \sqrt{\frac{z^{2}}{4} /\left(1+\frac{z^{2}}{12}\right)}$.

At expansion by powers $z=\tau \omega$, we have $\varphi=z\left(1+\bar{r}_{2} z^{4}+O\left(z^{6}\right)\right)$, i.e.

$$
\delta \omega=1+\bar{r}_{2} \omega^{4} \tau^{4}+O\left(\omega^{6} \tau^{6}\right), \bar{r}_{2}=7 / 1152=0.00608 .
$$

So, the Numerov's scheme has for the test equation the same order of accuracy. Let us compare coefficients at major term of the error $-\omega^{4} \tau^{4}$. For instance, for the scheme (5) at $\gamma=1 / 12, \alpha=1 / 8, \beta=1 / 24$, we have $r_{2}=(\beta-6 \alpha \gamma+1 / 40) / 12=0.00035$. So, the error for the Numerov's scheme is $\bar{r}_{2} / r_{2} \approx 17$ times higher compared to that for the scheme (5), (6). Besides that, stability condition $\mu \leqslant 4$ leads to restriction for the step by time $\tau^{2} \leqslant 6 / \omega^{2}$ that is more rigid compared to the schemes named above.

Even worse is accuracy of a common scheme with second order of accuracy (Samarskii, 1977)

$$
D y_{\bar{t} t}+A y=\varphi .
$$

Since for it $\delta \omega=1+r_{1} z^{2}+O\left(z^{4}\right)$, where $r_{1}=1 / 12=0.083333$.

\section{Conclusion}

Thus, this paper presents a method of high accuracy solution of boundary value problem for the equation of internal waves of a stratified fluid. The estimates of precision of the method with sufficient smoothness of the solution of the differential problem. On the basis of analysis of variance shows the benefits of the scheme (5), (6) over the known schemes, in particular, the Numerov scheme (12) and second-order accuracy scheme (14). Using the interpolation representation of the solution (6), if necessary, to obtain a solution and its derivative at any point in time. Numerical implementation of the scheme (5), (6) given in (Moskalkov \& Utebaev, 2011b). 


\section{References}

Bakhvalov, N. S., Zhidkov, N. P., \& Kobelkov, G. M. (1987). Numerical Methods. Moscow: Nauka.

Declu, J. (1976). Finite Element Method. Moscow: Mir.

Gabov, S. A., \& Sveshnikov, A. G. (1990). Linear Problems in the Theory of Non Steady State Internal Waves. Moscow: Nauka.

Moskal'kov, M. N. (1980). Scheme of the High Accuracy Finite Element Method for Solving Non Steady State Second Order Equations. Differential Equations, 16(7), 1283-1292.

Moskalkov, M. N., \& Utebaev, D. (2005). Investigation of Difference Schemes of Finite Element Method for Second Order Unsteady State Equations. Journal Computation Applied Mathematics, 92(1), 70-76.

Moskalkov, M. N., \& Utebaev, D. (2011a). Convergence of the Finite Element Scheme for the Equation of Internal Waves. Cybernetics and Systems Analysis, 47(3), 459-465. http://dx.doi.org/10.1007/s10559-011-9327-1

Moskalkov, M. N., \& Utebaev, D. (2011b). Comparison of Methods for Solving the Internal Wave Propagation Problem in a Weakly Stratified Fluid. Mathematical Models and Computer Simulations, 3(2), 264-271. http://dx.doi.org/10.1134/S2070048211020086

Quarteroni, A., \& Valli, A. (1994). Numerical Approximation of Partial Differential Equations. Berlin: SpringerVerlag.

Samarskii, A. A. (1977). Theory of Difference Schemes. Moscow: Nauka. 